\title{
Original
}

\section{Necesidades y actitudes de los facultativos de Atención Primaria frente a la gestión de las enfermedades profesionales}

\section{Needs and Attitudes of Primary Care Physicians coping with Administrative Management of Occupational Diseases}

\section{M. ${ }^{a}$ Josefa Ruiz-Figueroa ' , Matilde Fernández-Cid ${ }^{2}, M .{ }^{a}$ Fe Gamo-González ${ }^{3}$, Jordi Delclós-Clanchet ${ }^{4}$}

1. Área de Prevención de Riesgos Laborales. Subdirección Gral. de Recursos Humanos. Ministerio de Sanidad, Servicios Sociales e lgualdad. Madrid. España.

2. Sección Departamental de Sociología. Facultad de Ciencias Económicas y Empresariales. Universidad Complutense de Madrid. España.

3. Jefatura de Estudios. Escuela Nacional de Medicina del Trabajo del Instituto de Salud Carlos III. Madrid. España.

4. The University of Texas School of Public Health, Houston. Centro de Investigación en Salud Laboral (CiSAL), Universidad Pompeu Fabra, Barcelona. España.

\section{Recibido: 30-07-13}

Aceptado: 09-10-13

\author{
Correspondencia \\ María Fe Gamo González \\ Avda. Monforte de Lemos, 5 \\ 28029 Madrid. España. \\ Telf: 918224019 \\ Correo electrónico: mfgamo@isciii.es
}

Este trabajo está basado en el desarrollo de un curso online sobre el campus electrónico de la Escuela Nacional de Medicina del Trabajo (ISCIII) y con la financiación para su diseño por el Ministerio de Sanidad y Consumo y para su implementación por parte de dicho Ministerio y el Instituto Sindical de Trabajo, Ambiente y Salud (ISTAS)

Resumen

Objetivos: Desde 2007 la normativa relativa a la gestión por la Seguridad Social de las Enfermedades Profesionales y al sistema de información para su vigilancia epidemiológica, asigna a los médicos en Atención Primaria un papel como comunicadores a partir de su "sospecha diagnóstica". Este trabajo pretende mostrar algunas de sus necesidades expresadas en relación al conocimiento específico y sus actitudes y sensibilización en su práctica cotidiana respecto al origen laboral de estas patologías.

Métodos: Para ello, hemos analizado los textos generados en la interacción entre los alumnos y tutores participantes en 2 ediciones de un curso online ("Enfermedades Profesionales para Médicos de Atención Primaria”) realizado sobre el campus electrónico de la Escuela Nacional de Medicina del Trabajo del Instituto de Salud Carlos III, promovido y financiado por el Ministerio de Sanidad, entre otros.

Resultados: Los participantes expresan su inquietud en relación con: i) su falta de conocimientos específicos, ii) la carencia de utilidades o instrumentos habilitados en las historias clínicas informatizadas para orientarles en el diagnóstico de procesos "presumiblemente" laborales, y iii) los problemas de comunicación entre los actores del sistema.

Conclusiones: Destacamos su interés por saber cómo establecer el origen laboral de algunos procesos en un sentido amplio, más allá de la determinación de Enfermedad Profesional, y cómo apuntan soluciones que contemplarían: aumentar sus conocimientos, facilitarles el asesoramiento por expertos, hacer más visible lo laboral en la historia clínica de atención primaria y habilitar canales de comunicación eficaces con el resto de implicados en la gestión de las enfermedades profesionales. 
Palabras clave: Enfermedad Profesional; Contingencia Profesional; Sistemas de Información Sanitaria; Atención Primaria; Metodología Cualitativa.

Abstract

Goals: Since 2007 Spanish legislation mandates primary care physicians to report suspected occupational diseases for the purpose of occupational disease surveillance by the Social Security system. This paper presents the sensibility, attitudes and knowledge needs they express about the occupational origin of these conditions.

Metbods: We analyzed texts and commentaries generated by students and teachers who joined two different editions of an online course, "Occupational Diseases for Primary Care Physicians", developed on electronic campus of the National School of Occupational Health (Institute of Health Carlos III) promoted and funded by the Ministry of Health, among others.

Results: Participants expressed concerns regarding to: i) their lack of specific knowledge, ii) the absence of items in electronic medical records that could facilitate the detection of possible work-related pathologies; and iii) limited communication among the various stakeholders.

Conclusions: Primary care physicians expressed interest in learning more about how to establish the work-relatedness of disease, and offer suggestions aimed at improving their fund of knowledge, facilitating their access to expert consultation, highlighting the occupational exposure component of the medical history in primary care, and improving communication among all stakeholders.

Med Segur Trab (Internet) 2013; 59 (233) 393-404

Key words: Occupational diseases; Insurance, Work-related Conditions; Health Information Systems; Primary Health Care; Qualitative Research. 


\section{INTRODUCCIÓN}

La siniestralidad laboral, incluyendo accidentes de trabajo (AT) y enfermedades profesionales (EP), es un buen indicador del desarrollo preventivo de un país. En España, el sistema de información para los AT comenzó en 1900 y está bastante consolidado. Sin embargo, el sistema para las EP no funcionaba eficazmente y, en ese sentido, el Real Decreto 1299/2006 ${ }^{1}$ publicado en 2006 introdujo varias modificaciones: se actualizó el listado de diagnósticos y riesgos, se informatizó el proceso de notificación y se modificó la función en el proceso de los actores implicados en el sistema. Como consecuencia, desde entonces, una variedad de profesionales sanitarios (en las empresas, en las entidades aseguradoras y del Sistema Nacional de Salud) están obligados a iniciar el procedimiento de comunicación cuando sospechen que el proceso diagnosticado es profesional. La asignación de esa función a cualquier facultativo del Sistema Nacional de Salud (SNS) supuso una novedad sustancial que abría el campo más allá de la empresa y del sistema de aseguramiento. Se otorgaba así a los profesionales del SNS un papel "fundamental", entendiendo que muchas EP se presentan como enfermedades comunes o síntomas inespecíficos, y la mayoría de esos pacientes acuden inicialmente a médicos no especializados en medicina del trabajo, muy a menudo a su médico de familia y se espera de ellos que: sospechen, indaguen, deriven, propongan el estudio para la determinación de contingencia profesional, que informen adecuadamente a los pacientes y que consulten, en su caso, con la Inspección Médica ${ }^{2}$.

Sin embargo, ya desde los primeros momentos y desde diferentes ámbitos, se anticiparon dificultades: la norma supondría un nuevo problema para estos profesionales abrumados por la escasez de tiempo, y, en general, los médicos del SNS, y más específicamente los de Atención Primaria (AP), adolecían de falta de formación y/o experiencia específica en este tipo de patologías. Para afrontar esta situación desde el propio colectivo de atención primaria se ha planteado la necesidad de mejorar su formación, de simplificar la gestión de la incapacidad temporal por enfermedad profesional e, incluso, poder contar con un consultor experto en salud laboral, como vías de solución alternativas $^{2,3}$.

Nuestro trabajo sintetiza la expresión de los médicos de Atención Primaria acerca de sus necesidades de conocimientos y recursos específicos, y de sus actitudes y sensibilización en la práctica cotidiana respecto al origen laboral de estas patologías, para poder conocer si se está facilitando el desarrollo real del papel que en relación con las enfermedades profesionales les asigna la normativa vigente.

\section{MATERIAL Y MÉTODO}

Hemos contado con el material derivado de un programa formativo que dentro del marco del desarrollo de la Estrategia Española de Seguridad y Salud en el Trabajo (2007-2012), el Ministerio de Sanidad y Consumo encomendó ${ }^{4}$ a la Escuela Nacional de Medicina del Trabajo (ENMT), del Instituto de Salud Carlos III. Se trató de un curso a distancia sobre las EP contempladas en el RD 1299/06, dirigido a médicos de atención primaria (MAP), elaborado gracias al trabajo colaborativo de las Escuelas Profesionales de Medicina del Trabajo y de sociedades de medicina del trabajo y de medicina familiar y comunitaria, y dirigido a los profesionales del conjunto del Estado sobre plataforma electrónica (en formato online). La versión final incluye 13 unidades temáticas, agrupadas en 3 áreas: a) Fundamentos -historia clínica laboral y determinación de la contingencia profesional-, b) Normativa, y c) Clínica, orientada hacia la sintomatología que motiva la consulta. El curso, "Enfermedades Profesionales para Médicos de Atención Primaria", fue financiado por el propio Ministerio (en las 2 ediciones desarrolladas) y por el Instituto Sindical de Trabajo, Ambiente y Salud (la $2^{a}$ edición) y apoyado por las Comunidades Autónomas (CCAA) y las sociedades científicas colaboradoras. 
El curso se repitió en dos ediciones desarrolladas en 2011 sobre el campus electrónico de la ENMT. Cada semana del curso los alumnos accedían al material de una Unidad Temática y eran tutelados a través de las utilidades de comunicación de la plataforma: correos electrónicos interpersonales y la creación por los participantes, espontánea y voluntariamente, de Foros abiertos al conjunto de los participantes.

Entre las 2 ediciones completaron el curso 160 médicos que ejercen, en su mayoría, en la atención primaria o en unidades administrativas. La Figura 1 resume el número de alumnos que solicitaron $(n=299)$, iniciaron $(n=188)$ y completaron $(n=160)$ el curso.

Figura 1. Distribución de alumnos según Comunidad Autónoma de procedencia, ordenadas según el número total de diplomados

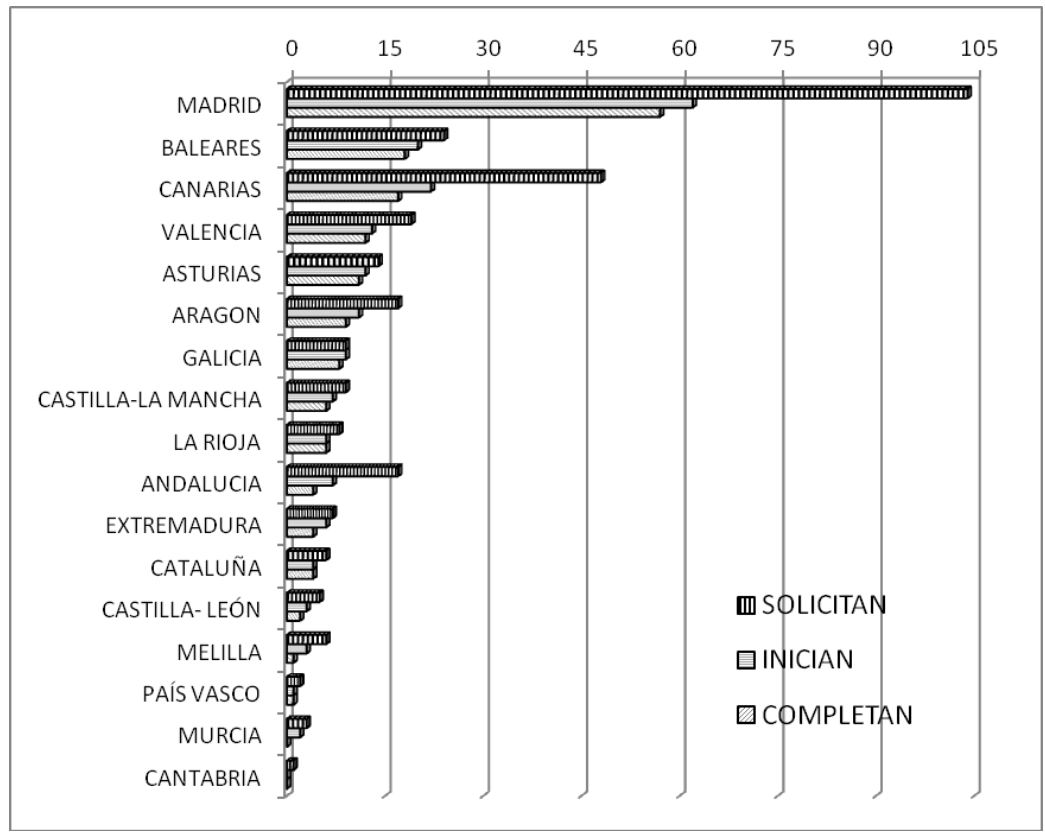

Las consultas y aportaciones de los alumnos participantes, en continua interrelación entre ellos y con los tutores, produjo unos textos que han sustentado el análisis que se presenta en este trabajo a partir de la información procedente de los debates abiertos en los Foros (Anexo 1) "para las Presentaciones" y "Temático", activas herramientas dialógicas de trabajo, insertas en la dinámica docente del Curso, donde los participantes preguntaban, interpelaban e intercambiaban experiencias en torno a las unidades temáticas. Para ello hemos revisado los materiales generados ${ }^{5}$ con relación a la Unidad Temática 1, "Historia clínica laboral y manejo clínico de la enfermedad profesional en atención primaria. Criterios de calificación y de relación causal de las enfermedades profesionales". 
Anexo 1. Descripción de la función y contenido de los Foros Para las Presentaciones y Temáticos

- En el Foro para las presentaciones, es donde los alumnos se presentan y comentan su experiencia e interés con relación al curso y a la salud laboral. Además, en este foro, para presentaros tenéis la opción de completar vuestro PERFIL como participante -cliqueando en el vínculo Perfil que está en la parte superior izquierda de la pantalla- al acceder a la pestaña de Editar Información y, allí, podéis contarnos lo que creáis conveniente compartir con el grupo de participantes. Por ejemplo, podéis explicar brevemente por qué estáis interesados en el tema del curso y qué esperáis de él. También podréis insertar una foto o cualquier imagen que nos sirva, de alguna manera, para 'vernos las caras'.

- En el Foro Temático los alumnos y tutores intercambiarán información sobre las UNIDADES TEMÁTICAS (dudas, consultas sobre los contenidos; propuestas y sugerencias para la práctica clínica). Es el lugar de encuentro de todos los que participamos en este curso, todos podremos abrir Temas de debate, incorporar materiales que estimamos de interés para el grupo de participantes, o presentar casos o experiencias profesionales relacionadas con los Temas que se van tratando en las Unidades Didácticas. Si el Tema está directamente relacionado con la Unidad activa de la semana, el tutor se encargará de motivarlo, moderarlo y mantener viva la comunicación. Pero, también es importante mantener el debate en los temas que no correspondan a la semana, porque a veces las nuevas Unidades nos van sugiriendo ideas que complementan o aclaran cuestiones abiertas en temas anteriores.

En el Foro Temático se abrieron 42 debates en la $1^{\mathrm{a}}$ edición y 28 en la $2^{\mathrm{a}}$ con referencia directa a esta unidad temática que fue tutelada, en las 2 ediciones, por uno de los autores (JD) y pretendía motivar a los alumnos del curso - "a propósito" de su instrumento de trabajo habitual- planteando que: "Si no se realiza una historia de exposición laboral, es poco probable que se llegue a un diagnóstico etiológico y si no se determina la exposición responsable de la sintomatología, dicha situación puede persistir o empeorary el tratamiento puede ser ineficaz" ". Además, 92 alumnos (66 de la $1^{\text {a }}$ edición y 26 de la $2^{\text {a }}$ ) hicieron aportaciones al Foro Para las Presentaciones que se incorporaron al análisis por ser las primeras expresiones de sus inquietudes hacia el tema.

La lectura que presentamos de este corpus textual prioriza la transcripción de la "voz" de los participantes, y su propuesta de explotación tiene como marco metodológico la perspectiva cualitativa de análisis conversacional en un contexto concreto. El texto producido transmite sentido, aunque no de forma literal: “...[sirviendo] de soporte para llegar a hacer visibles, e interpretables, las acciones significativas de los sujetos" Considerando que en el quehacer de los profesionales sanitarios entran en juego condicionantes del contexto, de sus vínculos con otros profesionales y estamentos, $y$, por supuesto, de la justificación y el sentido que tiene para ellos ${ }^{8}$, nuestra tarea ha consistido en seleccionar y organizar este material, y los resultados se sintetizan en 5 apartados o categorías temáticas que se presentan a continuación.

\section{RESULTADOS}

\section{Situaciones y procesos motivacionales para la realización del Curso}

A pesar de la pluralidad de situaciones de partida, resulta evidente que la decisión de apuntarse al Curso requiere cierta sensibilidad en torno al ámbito laboral, además de cierto conocimiento previo, incluso el reconocimiento de la carencia, de la falta de recursos -en un principio se subrayan los formativos- y la necesidad de mayor atención en este ámbito. La decisión de participar surge de diversas situaciones: desde la curiosidad, el interés por lo desconocido, hasta la necesidad de rectificación de prácticas erróneas, pasando por la evidencia de "huecos", aspectos no atendidos en el trabajo, que producen evidentes consecuencias negativas. (Véase Tabla I). 
Tabla I. Aportaciones de los participantes, en relación a "Situaciones y procesos motivacionales para la realización del Curso"

"Era consciente de mi desconocimiento sobre el tema de salud laboral/enfermedades profesionales y por eso me he apuntado al curso"

"La posibilidad de hacer un curso sobre enfermedades profesionales es algo que cuanto menos me llena de curiosidad, pues la verdad debo confesar no tengo mucha formación..."

"Creo importante tener la oportunidad de realizar este curso por la posibilidad de aplicación a nuestras consultas"

"... creo que va a serme muy útil en el trabajo en atención primaria..."

"Creo que no estaba haciendo bien las cosas, quizá porque aunque preguntes en qué trabaja un paciente desconoces los riesgos de su trabajo..."

"Ante un caso reciente de intoxicación aguda de uno de mis pacientes, he podido saber un poquito más de este tema, aunque reconozco que me faltó saber dónde buscar"

"Me he interesado por el curso porque no he tenido nunca formación sobre el tema durante todo el proceso de aprendizaje de nuestra profesión"

"Desde hace unos años soy tutora de los MIR [Médicos Internos y Residentes] en MFyC [Medicina Familiar y Comunitaria] y durante este tiempo y mientras yo fui residente no he visto un programa formativo específico en Salud Laboral. El conocimiento que adquieren los médicos residentes es a través de observación directa de su tutor en el periodo que rotan por el centro de salud. Además se oferta un curso optativo sobre 'Gestión de IT', en el que realmente no se tratan estos aspectos que estamos viendo en este curso (enfermedad profesional, normativa...) Creo que sería útil y necesario hacerlo de una forma más reglada y completa"

"Este Curso me interesa porque, en general, los médicos nos olvidamos que muchas de las enfermedades pueden tener su origen en una actividad laboral. Y actualmente, tal como está la situación económica, ni el mismo paciente ayuda a averiguarlo, ya que tener trabajo hoy es considerado una bendición"

"Me interesé por el curso para ver cómo podía integrar en la práctica clínica diaria el tema de las enfermedades profesionales... creo que los pacientes se merecen que se les trate según sus derechos; nosotros en AP [Atención Primaria] somos el eslabón de entrada a todo el sistema sanitario y no podemos tener esas grandes lagunas, como los diagnósticos de Enfermedades Profesionales, que en mi Comunidad son rara avis"

"Soy médico de AP en un Centro de Salud de una cuenca minera, no tuve relación con las E.P. [Enfermedades Profesionales], he diagnosticado pocas (supongo que habrán colado como enfermedad común)..."

"En la formación como residente de médico de familia no le dan importancia alguna a este tema..."

\section{Historia clínica y (des)información}

Con relación a lo que "provoca" los textos analizados, es decir, la historia clínica y laboral y la relación de los profesionales con esta herramienta en su práctica cotidiana, queda de manifiesto un primer y central problema relacionado con limitaciones burocráticas de partida: dificultades para localizar (en el formato electrónico) y cumplimentar la información sobre la situación e historia laboral del paciente-trabajador. Esta dificultad puede producir un importante "efecto eclipse" informativo ("no lo puedo poner", "no encuentro dónde") que derivaría en la imprecisión diagnóstica y/o la limitación en la atención. El problema se refuerza con la falta de formación en medicina laboral. Emerge, en el dialogo entre los participantes, una interesante reflexión/propuesta que, de forma radical, plantea que en la historia clínica la información relativa al trabajo pase desde un lugar secundario (cuando no oculto) a un lugar central. Esta crítica "radical" es contestada por una reflexión "realista" que sitúa en contexto de la práctica diaria y sus limitaciones el planteamiento de modelos "ideales". Se apuntan también las condiciones estructurales que impiden (más allá del deseo, la voluntad e incluso el "trabajo extra" de los profesionales) la puesta en práctica de la propuesta: la falta de tiempo en consulta, las carencias formativas, la limitación de recursos. La descripción del proceso en el trabajo concreto, que ofrecen varios miembros del foro, es ilustrativa de la dificultad de la tarea de incorporar en la práctica diaria la mirada exigida por la medicina del trabajo, y se agudiza cuando existen restricciones en el tiempo de consulta. A pesar de la conciencia de estos límites, se manifiestan propuestas de mejora. Por ejemplo, que se contemple la 
"historia laboral cronológica". Estos testimonios (Tabla II) se muestran muy ilustrativos del lugar que ocupa el trabajo en la historia clínica y de su minimización a priori como factor a valorar. Hay que añadir que frecuentemente la información alusiva al trabajo no "entra en materia", no alude a riesgos laborales ni a las condiciones de trabajo.

Tabla II. Aportaciones de los participantes, en relación a "Historia clínica y (des)información"

"Desde siempre me he encontrado con un porcentaje elevado de historias clínicas en atención primaria en las que no consta la ocupación, trabajos anteriores, etc., de cada paciente"

"(trabajo en un pueblo de Toledo como médico de familia) Cuando teníamos historias de papel, siempre registrábamos en la apertura la profesión del paciente, y todos sabíamos dónde encontrarlo. Ahora, con la historia electrónica... por curiosidad estoy mirando y con sorpresa descubro que ningún paciente tiene este espacio relleno..."

"Respecto a la toma de datos en la historia clínica, antes, en el formato de papel, había un campo para rellenar de accidente laboral o enfermedad profesional, que ha desaparecido en la historia informatizada, por lo que actualmente se tiende a pensar menos en ello"

"Sin duda no hay que dejar de soñar, pero mi duda se centra en la operatividad en la actualidad en España, con la organización de los CAP [Centros de Atención Primaria], la cultura social sobre la atención sanitaria, la actitud del profesional y los medios de los que disponemos (incluido el tiempo). [.....], si el médico no lo revisa es como si no estuviera, por lo que se requiere tiempo"

"Yo dejo una referencia en antecedentes personales capítulo de "sociales"

"Pues yo sí creo que es importante el lugar en qué se anotan los datos y sobre todo que todo el personal que trabaja con el paciente utilice el mismo lugar"

"No hay tiempo suficiente para que el paciente describa una jornada laboral... se me ocurre que se le podría dar un cuestionario para cubrir "fuera de la consulta" y aportarlo en sucesivas ocasiones... su estudio nos aportaría datos muy fiables y elementos de comparación"

"Siempre nos encontramos con la dichosa limitación del tiempo en las consultas para hacer historias adecuadas"

"Si en mi centro tenemos visitas telefónicas para comunicar resultados de analíticas en 1 minuto (incluyendo ver el informe, localizar al paciente y darle las explicaciones oportunas) no creo que dieran mucho más tiempo a la introducción de la vida laboral"

"Sería interesante considerar un apartado de ANTECEDENTES LABORALES, dentro del cuadro de ANAMNESIS"

"Trabajo para el SESCAM en Toledo y aquí utilizamos una aplicación informática llamada Turriano... te explico cómo se accede a ese apartado donde se refleja la profesión del paciente y que siempre encuentro vacío (sinceramente resulta engorroso de rellenar y difícil de consultar):

- accedemos a la historia del paciente; - cliqueamos hoja de antecedentes; - aparece una pantalla llena de pestañas (AP/AF/hábitos-sociosanitarios/hoja pediatría) y seleccionamos hábitos socio-sanitarios; nueva pantalla con estas opciones: nivel de instrucción/TRABAJO/estado civil/etnia/vive solo.

- si nos colocamos sobre trabajo se despliega esta información: asalariado sector privado, público, desempleado de larga duración, discontinuo, empresario, estudiante, labores domésticas, no procede, ocupación negocio familiar, otra situación, pensionista y además un espacio de texto libre para escribir lo que quieras...

.... y luego camina lo desandado para volver a la historia del paciente para iniciar un episodio y seguir trabajando"

“... mientras en OMI no haya un lugar para ello, sigo la máxima del área en la que trabajo: "si no está registrado, no existe", por lo que lo registro como parte de los datos generales de la anamnesis" 


\section{Señales, síntomas... y su vinculación al trabajo. Dificultades y pre-juicios limitantes}

Algunos profesionales manifiestan, ya en sus primeras aportaciones, una posición que asume la complejidad de establecer una relación de causalidad que derive en el reconocimiento de una EP. Apuntan, de forma connotada, que no se trata sólo de "elaborar una guía". La consulta constituye un microespacio de atención y en la valoración diagnóstica se deben considerar otros elementos que tienen relación con los contextos social, económico y profesional. La consideración -o no- del ámbito laboral como factor de salud está condicionada tanto por la normativa (del reconocimiento oficial de algunas enfermedades como "asociadas al trabajo") como por los prejuicios de los profesionales implicados, y resultan limitantes algunas asociaciones a priori.

A la dificultad de conocer los condicionantes laborales del paciente se suma la multiplicidad de conexiones entre trabajo y salud según se considere desde un punto de vista legal, científico, percibido por el paciente, o relativo a la "vida laboral" y no estrictamente a las condiciones de trabajo. En algunos casos descubren procesos que no están en el listado y no se consideran profesionales, aunque eso no esté negando un posible origen laboral. Les interesa especialmente la patología psíquica: estrés, patología depresiva o por trastornos adaptativos, que suponen una dificultad específica a la hora de establecer una conexión entre el trabajo y el riesgo, pero que son de especial relevancia en la práctica clínica (Tabla III).

Tabla III. Aportaciones de los participantes, en relación a "Señales, síntomas... y su vinculación al trabajo. Dificultades y pre-juicios limitantes"

"Según sea la patología que presente el paciente me acuerdo más de investigar en los posibles tóxicos laborales (piel, pulmonar, canceres...) o no (ginecología, digestivo...)"

"Voy a aportar mi granito de arena desde el punto de vista de mi actividad que es la urgencia. En este ámbito estamos bastante concienciados en cuanto a los accidentes laborales, con el fin de encaminar a los pacientes a las Mutuas; no así en las enfermedades profesionales en las que, en mi opinión, pensamos poco"

"No he sabido encontrar contacto con sustancias capaces de producir un episodio agudo de manía. De todas formas el riesgo laboral no es solo físico sino también psíquico y la exposición a situaciones estresantes en el trabajo puede favorecer la aparición de un brote en los cuadros maniacodepresivos"

"Dentro de los trastornos mentales con incapacidad temporal, más del $75 \%$ se deben a 2 procesos: depresión y trastornos de la ansiedad"

“... referente a la etiología del paciente con manifestaciones de fase maniaca, puede haber sustancias químicas que las causen pero no hay que descartar el aspecto emocional en el trabajo con las repercusiones que tiene en nuestra salud"

"Existe la tendencia(a mi parecer) de preguntar por la ocupación según la edad del paciente, con lo que es posible que estemos omitiendo la exposición fuera del ámbito laboral y en personas que ya no son trabajadores activos"

"Voy a hacer una aportación: PARO, ¿hay alguno que lo apunte en la historia? ¿se estudia su impacto en la salud y por tanto en la salud laboral? Hay 3 subapartados: 1.- Situación de paro. 2.- Impacto al dejar el paro (las lesiones por sobreesfuerzo están a la orden del día entre el personal que ocupa puestos eventuales iy las enfermedades? Acuden a urgencias pero no a su médico, tienen que trabajar como sea). 3.-Impacto al dejar el trabajo (no sólo hay depresiones) y ¿a cargo de quién va?"

"Me parece muy interesante el curso porque he tenido múltiples dudas... Además supongo que esto se puede reagudizar con la crisis económica y la precariedad laboral. Atiendo a una población con alto grado de precariedad económica, laboral, y con alto porcentaje de inmigrantes"

"En muchas ocasiones los trabajadores desconocen que trabajan con elementos que pueden dañar su salud y no pueden identificarlos, con lo que se dificulta la indagación. Y si lo conocen, sobre todo en estos momentos de crisis, a veces no quieren que se declare por miedo a perder su empleo". 
Otro "prejuicio" que limita la perspectiva es el que alude a la edad "laboral", que tiende a eliminar aquellos pacientes "fuera" del margen etario de la vida laboral. En el mismo sentido, algún profesional manifiesta la importancia de atender, como elemento que vincula vida laboral y salud, la propia situación de paro, hoy cuestión central para buena parte de trabajadores/as, incluso cuando esta situación se produzca en términos periódicos y/o acotados (progresivas entradas y salidas del mundo laboral).

\section{El papel del MAP en la gestión de la enfermedad profesional}

Los participantes hacen explícita la necesidad de coadyuvar en la "emergencia" o visibilidad de estas carencias (formativas, instrumentales) y plantean alternativas orientadas a la inclusión de la información. Como primer paso, expresan su interés por "aprender a hacer las preguntas correctas y las gestiones adecuadas". La crítica a los formatos de historia clínica y las propuestas de recogida detallada de las condiciones de trabajo, resultan una sugerente aportación que toca también el papel del médico no sólo en relación al diagnóstico y el tratamiento (la "curación") sino también al seguimiento, el vínculo y la atención personalizada (el "cuidado").

En ocasiones manifiestan desigualdades provenientes de los diversos contextos espaciales (hábitat) de trabajo, que conllevan sus propias oportunidades y limitaciones. Alguien habla desde un pequeño espacio "rural" del conocimiento de su población, pero también de la necesidad de hacer visible el problema (si un hecho no se percibe, se actúa como si no existiera) y dejar constancia escrita de esta información (Tabla IV).

Tabla IV. Aportaciones de los participantes, en relación a "El papel del MAP en la gestión de la enfermedad profesional"

\footnotetext{
“... es cierto que en atención primaria, aunque se ven muchas patologías relacionadas con el trabajo,
} se enfocan en pocas ocasiones desde el punto de vista profesional"

"Espero poder en este curso aprender algo más de un tema prácticamente inexistente en nuestra formación, porque los casos de sospecha de exposición/enfermedad/riesgo profesional pasan a ser 'visibles' cuando aprendes a hacer las preguntas correctas y las gestiones adecuadas"

"En Cataluña estamos trabajando en la introducción de un sistema de alerta cuando aparecen ciertos diagnósticos, vistos en atención primaria, y que podrían (en algunos casos) tener un origen laboral"

"Llevo 22 años en el mismo puesto de trabajo y conozco bastante bien a la población. Sé de qué trabajan y de qué han trabajado mis pacientes (en general, hay algunos con cambios de empleo frecuentes que no consigo recordar), pero nunca lo he anotado en su historia. No sé si por falta de existencia de apartado específico en la historia informática o por prisa... Pero a partir de ahora reflejaré los datos en lugar visible (tipo 'condicionantes y problemas', 'antecedentes' o algo así) para que la información que yo conozco pueda estar accesible a otros compañeros"

"Yo soy un médico del trabajo aburrido de pelear con las Mutuas, lidiando al mismo tiempo con la empresa, trabajador y Seguridad Social. Harto de ser presionado por las empresas para su propio beneficio y harto de pelear en todos los bandos (empresas, Servicios de prevención, Mutuas). Por eso al final me vine a la atención primaria, en la que nuestra pelea es que no nos cuelen enfermedades profesionales ni accidentes de trabajo como patología común..."

"Desde luego la colaboración entre los participantes en el proceso de identificación, estudio, diagnóstico y tratamiento de la enfermedad profesional no solo va a enriquecer a todos sino que le facilitará al trabajador y al médico la gestión de la contingencia”.

\section{Problemas de comunicación}

Ponen en evidencia que la falta de comunicación entre las distintas partes involucradas en la detección, notificación y reconocimiento de la patología laboral ha originado una estructura inadecuada para la comunicación efectiva y real. Es de destacar que, en la 
práctica, todavía no se ha planteado de manera eficaz la relación entre los MAP y los de empresas o entidades aseguradoras. (Tabla V).

Tabla V. Aportaciones de los participantes, en relación a "Problemas de comunicación"

"Estas preguntas puede que no tengan respuestas claras y contundentes al día de hoy. Pero lo que es seguro es que hay que empezar a plantearlas, a planteárnoslas, y a planteárselas a los gestores"

"Es difícil la comunicación entre dos ámbitos (dos 'mundos') que son el sanitario y el laboral que, a estos efectos, tienen mucho que decirse y que, sin embargo, no se hablan o no se encuentran. Me refiero, entre otras cosas, a que si pretendemos trasladar los criterios científicos (o médicos) a los reconocimientos médico-laborales 'simplemente', o directamente, estaremos olvidando el contexto principal de los reconocimientos que es el mundo del trabajo y, asociado a ello, la legislación laboral... En fin, que desde mi experiencia tengo que decir que son dos 'mundos' mal comunicados, pero que irremediablemente vamos a tener que aprender un lenguaje común para entendernos y atendernos debidamente"

"Tengo la sensación de que los médicos de trabajo no interpretan los resultados de las pruebas en el contexto clínico del paciente. He tenido varios casos de bradicardias conocidas y estudiadas, pero sistemáticamente le sugieren al trabajador que acuda a su médico de atención primaria para valoración y estudio de su problema"

"Yo tampoco conozco ese "enlace" entre Primaria y Mutuas. De hecho en alguna ocasión que algún médico de la Mutua ha contactado conmigo (por teléfono) me he negado a darle información por no poder asegurarme la veracidad de su identidad ni su relación con empresa y paciente, y porque creo que la información debe ir a través de la Inspección (punto que la Inspección en algún momento ha asegurado). Otra cosa es que el trabajador te pida que emitas un informe y tú se lo des, y que luego él haga lo que estime oportuno. Tampoco he conseguido nunca cambiar una contingencia de común a laboral. Una vez que he emitido la baja como enfermedad común, la Mutua se desentiende, y tampoco sé cómo consignar en el sistema informático (aparte de en texto libre) que está en estudio para determinar si es laboral o no. ¿Alguien ha conseguido una vez emitida una baja como "E. común" que cambie a laboral?..."

"O sea que cada Comunidad Autónoma debería organizar su 'sistema de información' estableciendo el procedimiento (a quien comunicar, mediante qué sistema, con qué criterios, etc.) En la práctica creo que hay diversidad de situaciones, unas Comunidades tienen definido el "organismo competente" y organizado el sistema y otras, no. Por eso creo que lo mejor es, en cada Comunidad Autónoma, informarse sobre cómo está el tema, poniéndose en contacto con las unidades de salud laboral de las administraciones sanitarias (generalmente, con excepciones, están ubicadas en los órganos de salud pública de cada Comunidad, pues en buena lógica estas unidades deberían ser el "organismo competente" al que alude el RD.

"Por otro lado, independientemente de que esté establecido o no el sistema de notificación, creo que sería bueno que se produjeran contactos más a menudo entre los médicos de familia y los médicos de los servicios de prevención ante casos concretos y para esto, estas unidades de salud laboral de las administraciones sanitarias pueden hacer de mediadores: identificar el servicio de prevención de un trabajador concreto, poner en contacto a los médicos de los dos ámbitos (atención primaria y servicio de prevención), asesorar en cuanto a medidas preventivas a tomar, etc.]"

\section{DISCUSIÓN}

El seguimiento de este diálogo proporciona interesante información de un proceso que transita desde el motivo y decisión de realización del propio curso, pasando por las sugerencias e interrogantes que suscitan sus contenidos, hasta las posibilidades, límites, formatos y usos concretos de la historia clínica como herramienta de trabajo. Todo ello anclado en propias experiencias, marcos de intervención, condicionantes institucionales o alternativas y propuestas de mejora, con frecuencia sugeridas desde la práctica y el trabajo concreto y nos da pistas para conocer el lugar que el ámbito laboral ocupa entre 
estos profesionales. Es importante recordar que la mayor o menor consideración de la vida laboral desde la mirada clínica garantiza, o no, el cumplimiento del papel asignado por la normativa vigente sobre enfermedades profesionales.

Se entiende que los profesionales destaquen los límites ${ }^{9}$-en primer lugar formativos, pero no sólo- y la búsqueda de alternativas ${ }^{10}$ para la mejora en la atención, y se constata la disonancia entre las carencias y lo prescrito por la normativa, que reconoce que el proceso de notificación se produce "sin una vinculación suficiente con el profesional médico que tiene la competencia para calificar la contingencia o con aquel otro que pueda emitir un diagnóstico de sospecha...”.

Partíamos de la premisa de que la historia clínica, generalizadamente informatizada, constituye un instrumento para la actuación en la consulta de atención primaria, orientado a mantener su desempeño con un enfoque comunitario y global del paciente [personatrabajador] y que, como tal, debería conformarse como elemento facilitador para la detección y la notificación de estas patologías. Los resultados indican que debe mejorar la inclusión de la información laboral en la historia. Dos estudios recientes muestran que solo de un cuarto a un tercio de las historias clínicas, tanto en centros de AP como en pacientes ingresados, contenían una descripción de la ocupación del paciente. Aún más alarmante, cuando se comparó esta cumplimentación en formato informatizado con la era de la historia en papel, aquella descendió a un $8 \%$ porque en el diseño electrónico no se incluyó una casilla para recogerlo ${ }^{11,12}$.

La falta de tiempo en la consulta es una barrera importante. Sin embargo, no sería necesario que para cada paciente se cumplimentara una historia laboral completa, a revisar por el médico. Aunque eso sería ideal, no es realista. Se podrían encontrar formas más efectivas de obtener información laboral, sin que suponga una carga adicional de tiempo: por ejemplo, se podría usar el diagnóstico del médico para "forzar" una alerta que "orientara" al médico a preguntar por ocupaciones o exposiciones concretas, ligadas a ese diagnóstico. Es el objetivo que orienta las acciones iniciadas en varias CCAA con los sistemas de alerta laboral para patologías concretas (de una lista).

Es importante recordar que al MAP no se le pide establecer la relación causal con el trabajo de manera concluyente, sino que pueda pensar en y, consecuentemente, notificar la sospecha de una patología laboral. La inquietud por ampliar el campo de interés desde la enfermedad profesional a la salud laboral en conjunto, a su vez, ayudaría al médico a que "el trabajo esté en su cabeza" durante la consulta, paso previo necesario para la identificación y sospecha de la patología laboral.

En relación con la necesidad de coordinación y comunicación entre los elementos del sistema, se han ido desarrollando actuaciones en diferentes CCAA ${ }^{13-15}$, orientadas a la incardinación de estos diversos profesionales en el sistema: a) coordinación entre médicos, inspectores y entidades colaboradoras y gestoras, b) automatización del proceso, incorporando a la historia sistemas de alerta al detectar ciertos diagnósticos, o c) sistemas dirigidos a completar o cruzar los datos de casos sospechosos con las circunstancias de exposición laboral del paciente.

El análisis de los textos ha evidenciado algunas actitudes y necesidades de los MAP frente a su papel en la gestión de las EP. Destacamos que:

- Reconocen su falta de formación en medicina del trabajo (en general) y en enfermedades profesionales (en particular), en relación paradójica con la expectativa legal de que desarrollen unas competencias para las que no han sido formados. Y reclaman formación para cubrir estos aspectos deficitarios.

- Señalan la necesidad de asesoramiento en el manejo de patologías profesionales. Así disminuiría el temor a equivocarse en el diagnóstico y serviría también para saber cómo derivar con más argumentos a las mutuas y resto de implicados.

- Muestran interés en que las historias clínicas incluyan items específicos acerca del trabajo del paciente que podrían activarse mediante una alerta ligada al diagnóstico. Eso les orientaría en la anamnesis y serviría como herramienta de 
formación continua, siempre que fuese de acceso fácil y no añadiese una carga adicional a la consulta.

La asunción de esta tarea -incrementar formación y conciencia- constituye un problema organizativo que atañe a la planificación y ejecución del sistema de salud. Y apunta a los ejes alusivos (lo normativo/lo real/lo deseable) sobre los que se mueve este fenómeno, así como a la necesidad de mejorar la coordinación entre las diversas partes y niveles de acción del sistema.

\section{BIBLIOGRAFÍA}

1. Boletín Oficial del Estado. Real Decreto 1299/2006, de 10 de noviembre, por el que se aprueba el cuadro de enfermedades profesionales en el sistema de la Seguridad Social y se establecen criterios para su notificación y registro. BOE $n .^{\circ} 302$ de 19/12/2006.

2. López Romero A, León Vázquez F, Holgado Catalán MS. Editorial. Enfermedad profesional y médicos de familia. Med Segur Trab (Internet) 2010; 56 (219): 109-113.

3. López Gandía, JL; Agudo Díaz, J. Nueva Regulación de las Enfermedades Profesionales. 4ª ed. Albacete: Fraternidad-Editorial Bomarzo, 2007.

4. Boletín Oficial del Estado. RESOlUCIÓN de 5 de octubre de 2007, de la Secretaría General de Sanidad, por la que se publica el Acuerdo de encomienda de gestión entre el Ministerio de Sanidad y Consumo y el Instituto de Salud Carlos III para la colaboración y asesoría científico-técnica en materia de las enfermedades profesionales. BOE n. ${ }^{\circ} 257$ de 26/octubre/2007.

5. Gordo López A. Análisis del Discurso: los jóvenes y las tecnologías sociales. En: Gordo López A, Serrano Pascual A., coord. Estrategias y prácticas cualitativas de investigación social. Madrid: Pearson Educación, s.a.; 2008.

6. Delclós J, Serra C. UNIDAD TEMÁTICA 1. HISTORIA CLÍNICA LABORAL Y MANEJO CLÍNICO DE LA ENFERMEDAD PROFESIONAL EN ATENCIÓN PRIMARIA. CRITERIOS DE CALIFICACIÓN Y DE RELACIÓN CAUSAL DE LAS ENFERMEDADES PROFESIONALES. Curso "Enfermedades Profesionales para Médicos de Atención Primaria" MSPS/ ENMT-ISCIII. Campus electrónico ENMT, 2011.

7. Alonso Benito LE. La mirada cualitativa en sociología. $1^{a}$ ed. Madrid: Editorial Fundamentos, 1998.

8. Calderón C. Criterios de calidad en la Investigación Cualitativa en Salud (ICS): apuntes para un debate necesario. Rev. Esp. Salud Pública. 2002; 76 (5):473-482.

9. Santibáñez Margüello M, Alonso Echabea E, Tamayo Medel G, Bolumar Montrull F, Vioque López J. Percepción del personal médico de atención primaria de salud acerca de sus funciones, formación y conocimientos en materia de salud laboral. Aten Primaria. 2008;40(1):7-14.

10. Cortès Franch I. COMENTARIO EDITORIAL La atención primaria de salud, un agente clave de la salud laboral. Aten Primaria. 2008;40(1):14-16.

11. Ditolvi Vera G, Benavides FG, Armengol O, Barrionuevo-Rosas L. CARTA AL DIRECTOR. Cumplimentación de la ocupación en las historias clínicas de la Atención Primaria: 1992 - 2007. Aten Primaria. 2010;42(9):486-491.

12. Aguilar Rodríguez AA, Ramada Rodilla JM, Delclós Clanchet J. Cumplimentación de la ocupación en las historias clínicas en un sistema hospitalario público: 2006-2010. Arch Prev Riesgos Labor 2012; 15 (3): 124-128.

13. Santolaria Bartolomé E, Esteban Buedo V, Casanova Vivas S. Guía para la comunicación de sospechas de enfermedades profesionales. Valencia: Generalitat. Conselleria de Salut, 2010.

14. Generalitat de Catalunya Departament de Salut. Institut Català d'Avaluacions Mèdiques. Instrucció $01 / 2007$ Valoració de la contingència profesional ICAM. Instrucció 01/2007.

15. Boletín Oficial del País Vasco. INSTRUCCIÓN n. ${ }^{\circ} 1$ de 26/12/07, sobre el procedimiento de comunicación de las Enfermedades que podrían ser calificadas como profesionales o cuyo origen profesional se sospecha.

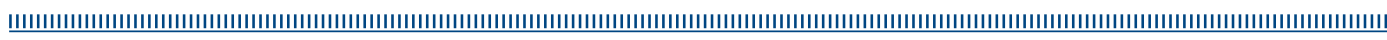

\section{AGRADECIMIENTOS}

A todos los profesionales que participaron en estos cursos con una dedicación tal que nos ha permitido acercarnos a ellos en una relación abierta y productiva, con el interés de mejorar no sólo nuestros conocimientos en salud laboral sino, sobre todo, la praxis concreta diaria en la consulta. 